\section{Ist Krebs für psychisch Kranke eher tödlich?}

\author{
Menschen mit psychischen Erkrankungen sterben häufiger an Krebs als \\ seelisch gesunde. Offenbar wird die Krebserkrankung bei ihnen nicht nur \\ später diagnostiziert, sondern auch weniger aggressiv behandelt.
}

$D^{i}$ ie Krebsletalität ist bei psychisch kranken Menschen etwa $30 \%$ höher als in der Allgemeinbevölkerung - die Krebsinzidenz ist in dieser Patientengruppe jedoch nicht erhöht. Die australische Arbeitsgruppe um Kisely et al. geben als Erklärung für das höhere Sterberisiko an: „Psychiatrische Patienten haben mit größerer Wahrscheinlichkeit bereits Metastasen, wenn der Krebs diagnostiziert wird, und sie haben schlechtere Chancen, eine spezialisierte Behandlung zu erhalten."

Die Mediziner verglichen mithilfe von Krebsdatenbanken die Allgemeinbevölkerung Westaustraliens mit Einwohnern, die eine psychiatrische Diagnose erhalten hatten. Letztere waren bei der Krebs- diagnosestellung im Mittel 64,3 Jahre alt, psychisch Gesunde dagegen 63,2 Jahre. Die altersbereinigte Krebsinzidenz war mit 368 versus 417 Fällen pro 100.000 Personenjahren bei Menschen mit psychischer Erkrankung sogar niedriger als in der Bevölkerung. $51 \%$ der psychisch kranken Krebspatienten starben im Studienzeitraum (1998 bis 2007). Die Mortalität lag bei den Männern um $52 \%$ und bei den Frauen um $29 \%$ über der von anderen Krebskranken. Auch wenn nur krebsbedingte Todesfälle berücksichtigt wurden, blieb die Sterberate um $20 \%$ erhöht.

Zum Zeitpunkt der Krebsdiagnose hatten $7,1 \%$ der Menschen mit psychischer Erkrankung versus 6,1\% in der Allgemein- bevölkerung bereits Metastasen entwickelt. Aber auch nach der Diagnose waren deutliche Differenzen in der Versorgung von psychiatrischen Patienten und der Bevölkerung insgesamt zu erkennen. Eine chirurgische Therapie wurde bei psychisch kranken Menschen mit einer um 19\% geringeren Wahrscheinlichkeit vorgenommen, insbesondere Karzinome des Kolorektums, der Brust und der Zervix wurden seltener operiert. Außerdem erhielten die Patienten weniger Chemotherapiesitzungen (im Mittel 10,3 vs. 11,4), und bei Darm- und Brustkrebs wurde seltener bestrahlt. „Offenbar ist psychiatrischen Patienten der Zugang zur Krebsversorgung erschwert, beziehungsweise diese bleibt oft hinter dem Standard zurück ", kritisieren Kisely et al.

bs

Kisely $S$ et al. Cancer-Related Mortality in People With Mental Illness. Arch Gen Psychiatry 2012; Epub Dezember 2012, doi:10.1001/ jamapsychiatry.2013.278

\title{
Stört andere Pillenfarbe die Compliance?
}

\begin{abstract}
Erhalten Patienten plötzlich Antiepileptika von anderer Farbe oder Form, beeinträchtigt dies möglicherweise die Therapietreue. Einer mangelnden Adhärenz geht vermehrt ein Tablettenwechsel voraus.
\end{abstract}

B ei Epilepsiepatienten sollte man bei einem Wechsel zwischen wirkstoffgleichen Präparaten wegen des engen therapeutischen Fensters und der Unterschiede in der Bioverfügbarkeit vorsichtig sein. Für die Compliance könnten sogar die veränderte Form und Farbe einer Pille entscheidend sein.

US-amerikanische Forscher werteten in einer Fall-Kontroll-Studie Daten von über 60.000 Patienten mit einer Antiepi-

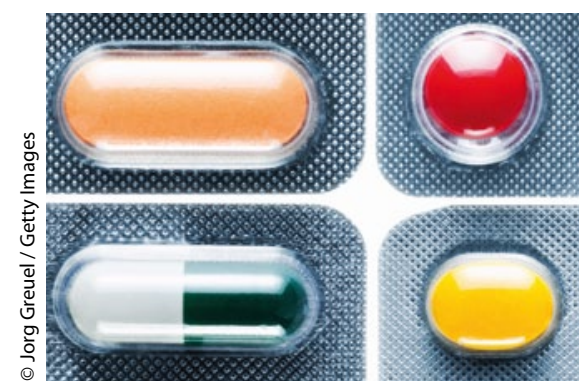

Welche wird regelmäßig genommen? leptikatherapie aus. $23 \%$ von ihnen waren an Epilepsie erkrankt, die übrigen litten an Schmerzen, affektiven oder anderen psychischen Störungen. Patienten, die ihr Rezept nicht innerhalb von fünf Tagen erneuerten, nachdem ihr Pillenvorrat hätte aufgebraucht sein müssen, wurden als nicht adhärent, solche, die sich stets rechtzeitig eine neue Packung verschreiben ließen, als adhärente Patienten eingestuft. Dann überprüften die Ärzte bei allen, ob es bei den zwei vorhergehenden Rezepten einen Wechsel zu einem Präparat mit anderer Form oder Farbe gekommen war.

Das Ergebnis: Bei 1,27\% der nicht adhärenten Patienten wurde ein Wechsel der Pillenfarbe bei den beiden vorausgegangenen Verschreibungen festgestellt, dagegen nur bei $0,97 \%$ der adhärenten Patienten. Relativ betrachtet war ein Wechsel der Farbe bei den nicht adhärenten Patienten damit um ein Viertel häufiger aufgetreten. In der Subgruppe der Epilepsie- kranken wurde ein Wechsel der Pillenfarbe bei 1,74\% mit Adhärenzproblemen und bei $1,16 \%$ ohne solche Probleme beobachtet (relativer Unterschied: $33 \%$ ).

Insgesamt darf allerdings auch bezweifelt werden, ob der Wechsel der Farbe tatsächlich einen deutlichen Einfluss auf die Compliance hat. Vielleicht verschrieben die Ärzte das neue Präparat zeitgleich mit einer Dosisreduktion, sodass die Packungen länger ausreichten. Darin sehen auch die Studienautoren eine mögliche Erklärung. Bedenkt man, dass nach Schätzungen mehr als $50 \%$ der Patienten ihre Medikation nicht vorschriftsmäßig einnehmen, dann dürften Schwankungen der Adhärenz von einem halben Prozent wohl kaum ins Gewicht fallen.

Nachvollziehbar ist sicherlich die Schlussfolgerung, dass man gerade bei Epilepsiepatienten mit einem Präparatewechsel vorsichtig sein sollte - dies gilt aber unabhängig von Form und Farbe der neuen Tabletten.

Thomas Müller

Kesselheim AS et al. Variations in Pill Appearance of Antiepileptic Drugs and the Risk of Nonadherence. Arch Intern Med, online 31. Dezember 2012; doi:10.1001/2013. jamainternmed.997 December 31, 2012 